\title{
Research on the Long-distance Transmission
}

\author{
Xueli Wu ${ }^{1}$, Xiuyuan Yang ${ }^{1}$, Hong Shen ${ }^{2}$, Qinyong Zhou ${ }^{2}$ \\ ${ }^{1}$ Shool of Automation Beijing Information Science and Technology University, Beijing 100192, China \\ ${ }^{2}$ China Electric Power Research Institute, Beijing 100192, China \\ Email: xueliwu369@163.com
}

Received March, 2013

\begin{abstract}
In view of the imbalanced distribution of power load and resources, including the status of "electric shortage" in some cities in our country, the article discusses the long-distance transmission technology. It mainly analyzed two ways of the long-distance transmission: UHV AC transmission and UHV DC transmission. The fractional frequency transmission technology and half wavelength AC transmission technology of AC transmission are introduced. Some key technologies of long-distance transmission are described. It has a guess for long-distance transmission future direction.
\end{abstract}

Keywords: Energy; Long-distance; Ultra-high Voltage (UHV); Alternating Current (AC); Direct Current (DC); Transmission

\section{Introduction}

According to the requirements of the development of China's national economy, the national power generation capacity of 1100-1200 GW plan is expected to be realized in 2020. China's current situation analysis, energy and electricity load in the geographical distribution of exeme imbalance, according to the latest data, about $68 \%$ of the hydropower resources in China located in the southwest region, about $76 \%$ of the coal resources disibution in the north or the northwest of China, and about $70 \%$ of the electricity load is mainly concentrated in the eastern coastal areas [1-5]. The situation is shown in Figure 1.

More and more serious with the consumption of resources, a lot of our region have been facing the risk of resource depletion, coal resources is an important resource for thermal power, coal shortage has caused

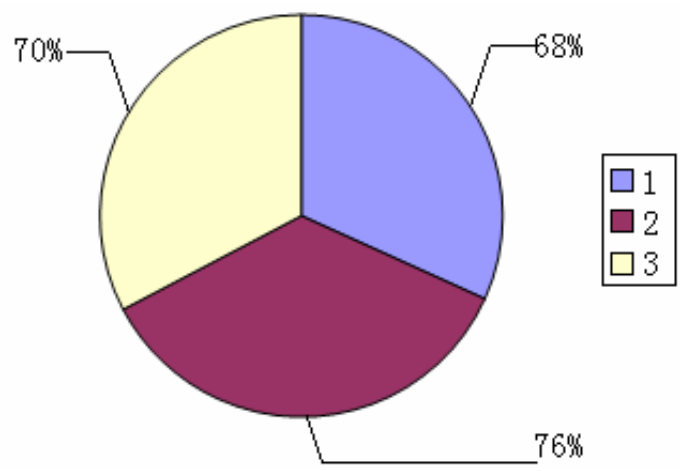

Figure 1. Resources and electricity load distribution. some of our cities face a "shortage" dilemma, serious impact has an affect of the residents' production and livg. But the development of nuclear power, wind power, hydropower and other new energy can significantly reduce the proportion of thermal power. As in Sweden, nuclear power and hydropower accounted for $70 \%$ of the proportion. And by the end of 2008, the installed capacity of wind power in China has more than 10 million kilowatts capacity. If this part of wind power transfer out through long-distance transmission, can not only solve the problem of "shortage", but also make a great contribution for scheme $\mathrm{s}$ of grid interconnection and "North to South".

As the adjusting of the strategic approach in our country, just like "development of the western region", "West to East", to promoting the implementation of the policy. Making large-capacity, and the imminent application of long-distance power transmission technology, highapacity, high reliability combined power system is bound to become the future development direction. Therefore, high pressure, long-distance power transmission technology has become an integral part of grid technology.

\section{Long-distance Transmission Mode}

According to the long distance transmission, in order to solve the long-range, high-capacity transmission process, the article put forward the UHV transmission technology. The transmission capacity of $500 \mathrm{kV}$ voltage level is about 1000-1500 MW, and the transmission distance is about 300-1000 km [5], then it's not able to fulfill the requirements of the grid no matter on the length, the cacity of the transmission, or limit the short-circuit current. 
Based on the issues above, in April 2005, the Chinese Society of Electrical Engineering, China Machinery Enterprise Confederation and the China Power Engineerg Consultants Group Corporation jointly organized discussion of UHV AC and DC standard voltage clear of AC UHV nominal voltage $1000 \mathrm{kV}$, the highest voltage for equipment $1100 \mathrm{kV}$; UHV DC rated voltage $800 \mathrm{kV}$ [3]. Paragraphs in the national network of UHV transmission grid transmission capacity of about 5-20 GW, conveying distance of about $600-2000 \mathrm{~km}$ as the choice.

\subsection{UHV AC Transmission Technology}

As the long-distance transmission with the long-distance and high-capacity, the State Grid Corporation has at the end of 2004 to accelerate the construction of UHV power grid in order to the $1000 \mathrm{kV}$ exchange system as the core of a strong national grid as a strategic objective. UHV AC transmission technology can solve the regional power grid interconnection trunk system increased trend of increase brought the system unstable and short-circuit level, reduce transmission losses and efficient transmission, This paper briefly describes AC Transmission Fractional Frequency Transmission and a half-wavelength AC transmission technology.

\subsubsection{Fractional Frequency Transmission Technology} In traditional power transfer process, generally used to change the voltage level of the way to achieve longdistance and large-capacity transmission, and subfrequency transmission technology is selected under the conditions of the lower frequency transmission by changing the frequency of the electrical energy in the transmission process, and at higher electricity frequency, i.e. the frequency of electrical energy in the control conveying process in order to improve the energy transfer due to the conveying distance is too large power loss and other problems.

Fractional Frequency Transmission diagram is shown in Figure 2. You can see from the chart that the frequency generator issue 50/3 $\mathrm{HZ}$ the electrical energy in the process of transmission keep 50/3 HZ frequency unchanged until the transmission end of the frequency multiplier frequency adjustment $50 \mathrm{HZ}$ frequency power system power supply to the Public. Compared with the traditional power transmission frequency with $50 \mathrm{HZ}$, the frequency 50/3 HZ theoretically line transmission capacity can reach three times that of the former, can

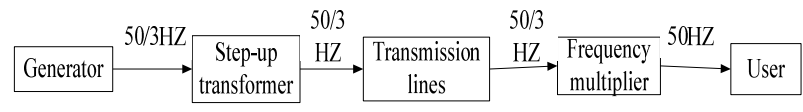

Figure 2. Fractional Frequency Transmission schematic diagram.

reduce the number of transmission circuits and an area of the corridor [6].

Xifan Wang, the professor of Xi'an Jiao tong University, pointed that the Hydro-generators speed is low, for giving low-frequency power, simply reduce the number of pole pairs. Hydro-generators can transformer core area by increasing the number of turns to achieve the decrease in energy frequency issued [9-10].

Fractional Frequency Transmission critical frequency multiplier, the double main control system is AC - AC inverter circuit, to join AC - AC converter, however, presents a lot of problems; the problem which usually appears is that the inverter thyristor guide passes to cause the failure. To solve this problem [7-8], analysis the literature on the single bridge and other bridge arm not conducting both cases for Fractional Frequency. According to the probably problem of transmission frequency converter for the divider, required to strengthen the developed of the frequency multiplier control system.

\subsubsection{Half-wavelength the AC transmission [11-13]}

The half-wavelength AC transmission refers to the transmission of electrical distance of nearly a half-wave power frequency, frequency $50 \mathrm{HZ}$ electrical energy situation for our workers, and the half-wave transmission distance of $3000 \mathrm{~km}$. With a high degree of social and economic development, can not deny that the future of electricity transmission is transnational and even intercontinental, that $3000 \mathrm{~km}$ ultra-long-range transmission technology has higher practicality.

The $3000 \mathrm{~km}$ distance of half wavelength transmission of natural distance, and not in the long-distance transmission process every time to ensure the transmission distance is precisely $3000 \mathrm{~km}$, when the transmission distance of more than or less than $3000 \mathrm{~km}$ manual tuning technology need to take the actual line is tuned into a half-wavelength . Currently used in two ways: to increase the series inductance and shunt capacitance, only increase the shunt capacitance. Wherein, in the same over-voltage conditions, and the latter can transmit more power, when a failure occurs at the same time, the power loss of the former is smaller than the latter, the transient stability of the latter is preferred.

Literature [11] starting from the half-wavelength transmission technology, transmission characteristics, and clarify the advantages of the transmission mode, and also pointed out that such a transmission line does not require installation of reactive power compensation equipment from multiple angles, the reactive power line capacitance issued by line own inductance consumption. The literature analysis means half-wavelength AC transmission failure and its inhibition may occur. Verify the validity of the transmission mode. The literature [12] of the half-wave AC Transmission economic argument, drawn UHV DC transmission, certain funds within the 
transmission capacity of 5000MW under the half wavelength AC Transmission economy better than $800 \mathrm{kv}$ HVDC transmission.

\subsection{UHV DC Transmission Technology}

DC transmission as the the UHV transmission of a form, the major powers of the world's electricity currently an important means to solve the high-voltage, high-capacity, long-distance transmission and grid interconnection. Compared to AC transmission, DC transmission, power generation exchange system and exchange system of electricity without synchronous operation at both ends of the exchange system can be run in accordance with their respective frequency and phase; DC transmission is a two-wire, all savings supplies, to reduce the loss of transmission lines, etc..

UHV DC transmission project a 10-year development plan, China has completed in 2011 and put into operation the Xiangjiaba - Shanghai $800 \mathrm{kV}$ construction. Jinping I and II power plant are expected to be completed in 2014, the two power plants to the East China $800 \mathrm{kV}$ power transmission level HVDC Transmission Project [2], the nominal voltage of UHV DC transmission $800 \mathrm{kV}$. UHV DC transmission project put into operation in China: three Chang, three Guang, three Hu Engineerings, have achieved good results.

HVDC following schematic is shown in Figure 3, the DC transmission converter station (the rectifier and inverter stations), DC line, AC side and the DC side power filter, reactive power compensation device, the DC reactor which the converter station is the heart of the DC transmission system, alternating current converted into direct current through the rectifier equipment delivered to the receiving end through a DC transmission line, and through the inverter DC reverse into AC input AC grid.

Advantages of UHV DC transmission technology:

1) Conserve supplies, direct current transmission is compared to the two-wire AC. Transmission can save 1/3 of the transmission material.

2) The AC system of generating end and the receiving end don't need to run simultaneously, the both ends of

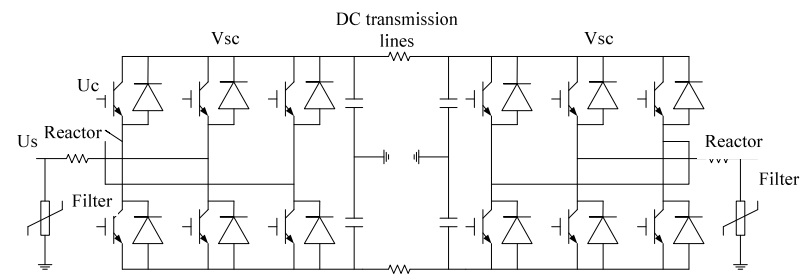

Figure 3. DC transmission schematic.

the AC system can be run in accordance with the respective frequency and phase, to facilitate adjustment.

3) With respect to the AC transmission compared to the pollution of the environment and the interference of the communication device.

\section{The Key Technical Issues of the Long-distance Transmission}

\subsection{Consolidated Potential Compensation Issues} [16]

Undertake the task of even the receiving end of the system, and long-distance power plant or a different partition power system long-distance transmission, longdistance transmission will cause some inconvenience to the power system, how the power system to maintain stability in the effective regulation has been is the key issue the integrated electric potential compensation technology proposed for this problem.

Integrated potential compensation by the string in the appropriate place of the long-distance transmission lines into an amplitude and phase can be adjusted continuously the potential to achieve a variety of functions needed to improve the power system operating characteristics. [15] verified by simulation analysis of the potential compensation in the transmission process, not only to improve the static stability of the system, while also able to reduce the power of the generator shaft imbalance, improve the transient characteristics.

\subsection{Overvoltage and Insulation Problems}

In the long-distance transmission process, due to the general use of UHV overhead lines transmission line to endure operating pressure, fault hazards, lightning impulse voltage is too high may cause problems, transmission lines insulation technology is a test. This insulation comprises two parts: the insulation and on the tower of the power transmission line of the insulator insulating. Insulation of transmission lines on the one hand to consider voltage requirements, on the other hand to consider the economic, select the correct and economical way to reduce the voltage level of the equipment is critical to the longdistance transmission. Domestic overhead line insulators porcelain insulators, three types of glass insulators and composite insulators and transmission line insulation, the choice of the insulator also equally important to be selected according to the specific circumstances of the transmission.

\subsection{Environmental Protection}

Long distance transmission of high-voltage, highcapacity network structure during operation on the inevitable existence of electromagnetic interference, radio waves, radiation, corona, and other environmental protection issues. 


\subsubsection{Electromagnetic Radiation}

Long-distance transmission of the electromagnetic radiation is mainly divided into two categories: active and passive jamming interference, an active interference mainly from the wires of the corona discharge and the corona current to the space radiation of electromagnetic waves, resulting in the increase of the background noise; passive interference including high-voltage transmission lines as great size metal grid to radio signals may produce secondary radiation, refraction, reflection and shielding.

Also, China is also an earthquake-prone geographical, the earthquake happened in Wen Chuan in 2008 has affected people by now, so the prediction and detection on earthquake are especially important. Seismic observation stations predict the activity of geological from the subtle changes of the geomagnetic field. Signals of magnetic field and DC magnetic field generated by DC transmission line are difficult to distinguish. If the current or parameter of the DC transmission line changed, the DC magnetic field will change too, which may cause interference to the geological staff, to generate the error signal.

\subsubsection{Corona Interference [14-17]}

Corona is decomposed into positively charged ions and negatively charged electrons due to the gas molecules in the air when the influence of a strong electric field, the field strength is further increased when the phenomenon of electron multiplier, which would form a corona discharge. Instant corona discharge generates a pulse current, the current energy in the form of electromagnetic waves outward release interested around the thus generated electromagnetic wave radio signal interference, but also affect the normal work of the high-frequency carrier channel. The instant of corona discharge will not only produce electromagnetic interference, also produces audio interference, irritability serious cause the surrounding residents.

\section{Development Prospects}

This paper introduces the research status of the longdistance transmission technology briefly, although the long-distance transmission are still some problems, but this is not deter its development and has been widely used in the current long-distance transmission technology. UHV long transmission technology also has improved steadily, with the forward to the continuous advancement of science and technology, some technical problems in the course of transmission will continue to be resolved, UHV transmission technology will have a good development. In the context of globalization, with unbalance of electricity load and the uneven distribution of resources of international or national, transnational and even intercontinental transmission may be achieved in future. Then there will be a major test on core technology of long-distance transmission.

\section{REFERENCES}

[1] Q. Y. Yuan, "Present State and Application Prospect of Ultra HVDC Transmission in China," Power System Technology, Vol. 29, No. 14, 2005, pp. 1-3.

[2] H. Feng and L. Xiao and F. S. Teng, "Integrative Voltage Compensation in Long Distance Transmission Lines," Power system technology, Vol. 23, No. 8, 1999, pp. 1-7.

[3] Research on Overview of China's UHV Transmission Technology, East China Electric Power, 2006, Vol. 34, No. 8, pp. 121-122.

[4] M. H. Zhu, "Energy Resourses, Integrated Interconnection Network, UHV Transmission,” High Voltage Engineering, Vol. 26, No. 2, 2000, pp. 28-30.

[5] J. X. Zhang, "Selection of UHV Voltage Levels and Transmission Line,” Journal of Qinghai University, Vol. 25, No. 1, 2007, pp. 14-18.

[6] X. F. Wang, C. J. Cao and Z. C. Zhou, "Experiment on Frational Frequency Transmission System,” Chinese Journal of Mechanical Engineering, Vol. 6, No. 2, 2005, pp. 6-12.

[7] Y. F. Teng, X. F. Wang and M. Deng, "Cycloconverter Non-conduction Fault and Related Problems in Fractional Frequency Transmission Systems," Power system automation, Vol. 32, No. 7, 2008, pp. 69-72.

[8] G. K. Li, H. F. Liang, C. Y. Zhao, M. Zhou and G. Y. Li, "Cycloconverter Non-conduction Fault and Related Problems in Fractional Frequency Transmission Systems," China Electric Power, 2004, Vol. 37, No. 4, pp. 43-48.

[9] X. F. Wang, "Study of Digital Simulation for Fractional Frequency Transmission System,” China Electric Power, 1995, Vol. 28, No. 3, pp. 8-12.

[10] X. F. Wang and X. L. Wang, "Feasibility Study of Fractional Frequency Transmission SYSTEM,” Power System Automation, Vol. 19, No. 4, 1995, pp. 5-13.

[11] G. Wang, X. C. Lv, Q. Q. Sun, Q. Y. Li and Q. M. Li, "Status Quo and Prospects of Half-wavelength Transmission Technology,” Vol. 34, No. 16, 2010, pp. 13-18.

[12] K. Sun, "Economic Analysis on UHV Half-wavelength AC Power Transmission," Power system technology, 2011, Vol. 35, No. 9.

[13] F. Lliceto and E. Cinieri, "Analysis of Half-wave Length Transmission Lines with Simulation of Corona Losses," IEEE Transactions on Power Delivery, 1988, Vol. 3, No. 4, pp. 2081-2091.doi:10.1109/61.194020

[14] W. M. Long, "The Environment Protection of Long Distance Transmission-Corona Interference," Guangdong power transmission technology, 2006, Vol. 2, pp. 12-15.

[15] S. Kumar, A. K. Mondal, H. Dieringa, et al., Analysing Hysteresis and Residual Strains in Thermal Cycling 
Curves of Short Fibre Reinforced. Mg- MMCs," Composites Science and Technology, 2004, Vol. 64, pp. 11791189. doi:10.1016/j.compscitech.2003.09.022

[16] S. Kumar, S. Ingle, H. Dieringa, et al., "Analysis of thermal cycling curves of short fibre reinforced MgMMCs," Composit eds Science and Technlogy, Vol. 63,
2003, pp. 1805-1814.

[17] H. Zhang, P. A. Anderson and G. S. Daehn, “Analysis of Thermally Induced Stress and Strain in Cont Inuous Fiber-reinforced Composite,” Metallurgical and Materials Transactions, Vol. 25, No. A, 1994, pp. 415- 425. 